\title{
Compact and low-loss asymmetrical multimode interference splitter for power monitoring applications
}

\author{
Andrea Zanzi, ${ }^{*}$ Antoine Brimont, Amadeu Griol, Pablo Sanchis, and Javier Marti \\ Valencia Nanophotonics Technology Center, Universitat Politècnica de València, València, Spain \\ ${ }^{*}$ Corresponding author: anzan@ntc.upv.es
}

Received 6 November 2015; revised 7 December 2015; accepted 7 December 2015; posted 8 December 2015 (Doc. ID 253437); published 4 January 2016

\begin{abstract}
This Letter presents a compact and low-loss $1 \times 2$ asymmetrical multimode interference (A-MMI) splitter in rib geometry for on-chip power monitoring at $1.55 \mu \mathrm{m}$, where a given alteration of the component cavity determines arbitrary values of the output power splitting ratios. The device shows reduced losses ( 0.4-0.8 dB) and robustness across a $40 \mathbf{~ n m}$ optical bandwidth (1540-1580 $\mathbf{n m})$. () 2016 Optical Society of America
\end{abstract}

OCIS codes: (040.6040) Silicon; (130.3120) Integrated optics devices; (230.1360) Beam splitters.

http://dx.doi.org/10.1364/OL.41.000227

Silicon-on insulator (SOI) is currently considered as one of the most promising platforms to achieve dense integration of photonic devices at low cost, owing to its high-index contrast and compatibility with mature complementary metal-oxide semiconductor (CMOS) fabrication process [1,2]. Multimode interference (MMI) devices are commonly used in modern photonics [3,4] and optoelectronic integrated circuits [5-7], owing to their compactness [8,9], large bandwidth, overall low losses $[10,11]$, and robustness against fabrication variations. As a result, their possible implementation as power splitters is very attractive [12]. Asymmetrical multimode interference (A-MMI) devices with strip configurations have been recently proposed [13]; however, to the best of our knowledge, no work on rib geometries has been reported yet. In this Letter, we present a compact and low-loss $1 \times 2$ MMI splitter featuring a rib geometry for on-chip power monitoring purposes, where an alteration of the cavity determines an efficient and stable splitting of the optical power.

The A-MMI has been designed in a shallow-etched waveguide configuration. To fulfill the compactness requirement and allow relaxed fabrication process tolerances, we have created a device with a multimode section $\left(L_{m m i} * W_{m m i}\right)$ of $10.5 \mu \mathrm{m} \times 3 \mu \mathrm{m}$, using 3D-FDTD simulations [14].

The schematic of the new proposed $1 \times 2$ A-MMI is sketched in Figs. 1(a)-1(d). The near infrared light is injected into the multimode region through a $450 \mathrm{~nm}$ wide waveguide and then tapered to reach a width of $0.85 \mu \mathrm{m}$. The two tapered output waveguides are precisely positioned at the first two folded images at $\pm W_{m m i} / 4$.

The tapers of the A-MMI are engineered to maximize the ingoing and outgoing light from the multimode region, and are symmetric and asymmetric at the input and outputs, respectively [Fig. 1(a)]. They have been modeled using 3D-FDTD simulations, and the optimized values for $\Delta_{1}$ and $\Delta_{2}$, respectively, are $0.2 \mu \mathrm{m}$ and $0.525 \mu \mathrm{m}$. The lengths of the input and output tapers, respectively, are $5 \mu \mathrm{m}$ and $15 \mu \mathrm{m}$. We chose to use asymmetric tapers at the outputs to avoid the presence of sharp corners on the device, reducing accordingly the losses caused by the roughness induced by the fabrication process. Moreover, this layout enables the coupling between the multimode region and the output waveguides to be maximized. In contrast with conventional symmetric $1 \times 2$ MMI featuring 50:50 power splitting ratios, our asymmetric MMI can achieve variable outputs splitting ratios through breaking the symmetry of the cavity.

The arbitrary power splitting ratios are obtained in the following manner: using as a starting vertex the connection point between the input taper and the multimode region, the cavity has been cut using the angle $(\theta)$ as a variable of reference as depicted in Fig. 1(a). The increment value of $\theta$, associated with an increasing removed area from the cavity, determines different values of the weaker output power ratio, denoted by $\mathrm{R}$, and the relative stronger output power ratio with a value of (1-R). Moreover, in the case of increasing values of $\theta$ (i.e., 15/85 A-MMI), the left-hand side of the weak output's taper $\left(\Delta^{*}\right)$ has to be reduced [Fig. 1(c)] to be consistent with the layout of the device.

We fabricated four different versions of the device, with four different values of R, namely, $15 \%, 25 \%, 30 \%$, and $40 \%$. This allowed us to evaluate the validity of our assumptions and simulations. The samples have been fabricated on standard siliconon-insulator (SOI) samples with a top silicon layer thickness of $220 \mathrm{~nm}$ (resistivity $\rho \sim 1-10 \Omega / \mathrm{cm}$ ) and a buried oxide layer thickness of $2 \mu \mathrm{m}$. To obtain the desired $70 \mathrm{~nm}$ depth etch [Fig. 1(b)] for both waveguides and grating couplers, the fabrication was performed with electron beam (RAITH 150), 
a)

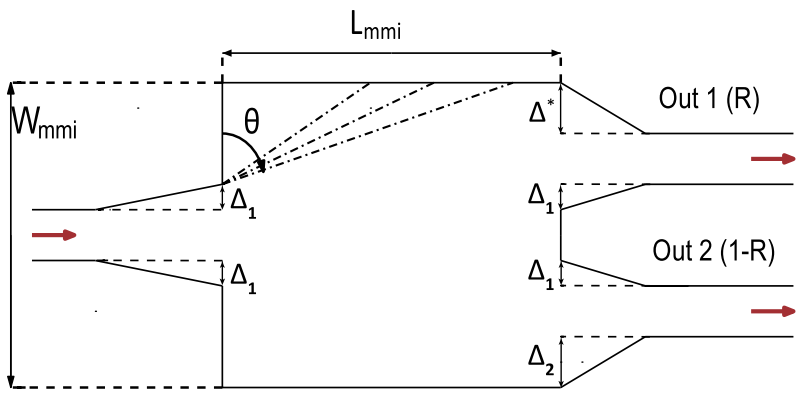

c)

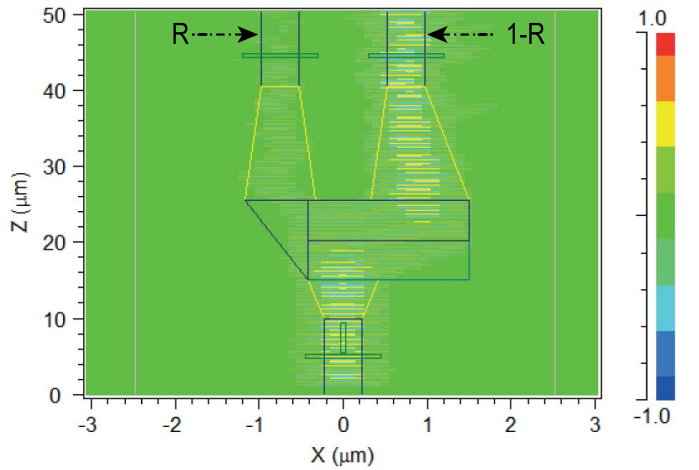

b)

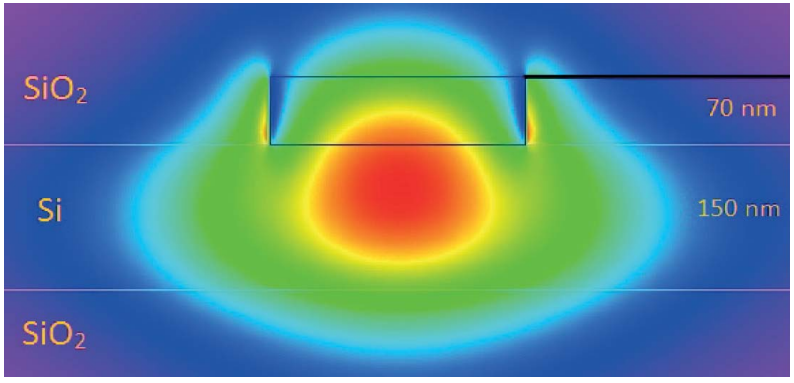

d)

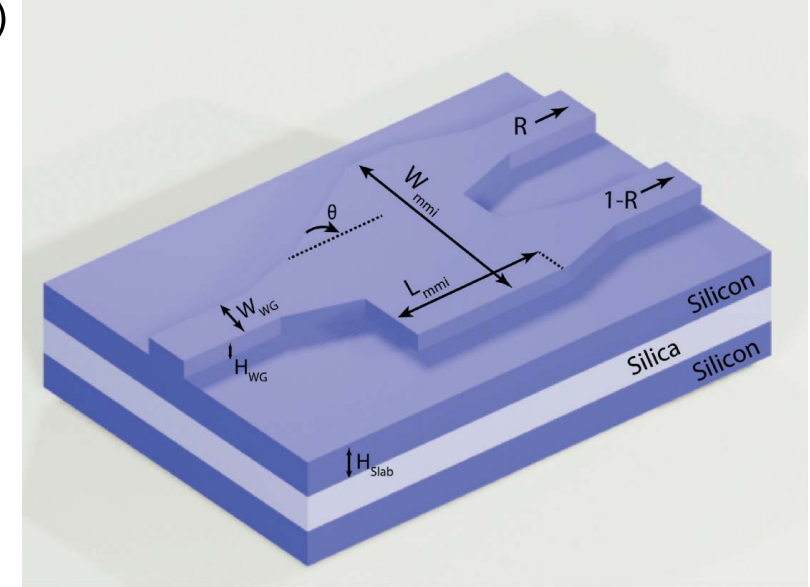

Fig. 1. (a) $2 \mathrm{D}$ schematic of a $1 \times 2 \mathrm{~A}-\mathrm{MMI}$ power splitter, which shows the design parameters. (b) Fundamental optical mode in a shallow etched configuration used to realize the device. (c) Distribution of the magnetic field $\left(H_{y}\right)$ in the multimode region of the asymmetrical MMI $(15 / 85$ A-MMI) $\left(\Delta^{*}=0.2 \mu \mathrm{m}\right)$. (d) 3D layout of the A-MMI. The SOI platform foresees the use of a $220 \mathrm{~nm}$ slab structure with $70 \mathrm{~nm}$ etch depth. All the splitters are designed using the following values: $L_{m m i}=10.5 \mu \mathrm{m}, W_{m m i}=3 \mu \mathrm{m}, W_{W G}=450 \mathrm{~nm}, \Delta_{1}=0.2 \mu \mathrm{m}$, and $\Delta_{2}=0.525 \mu \mathrm{m}$.

using a direct writing process performed on a coated $100 \mathrm{~nm}$ hydrogen silsesquioxane resist film. The process was optimized to reach the required dimensions employing an acceleration voltage of $30 \mathrm{keV}$ and an aperture size of $30 \mu \mathrm{m}$. After developing the HSQ resist using tetramethylammonium hydroxide as developer, the resist patterns were transferred into the SOI samples employing an also optimized "inductively coupled plasma-reactive ion etching" (ICP-RIE) process with fluoride gases. The shallow-etched passive silicon chip was then covered with $700 \mathrm{~nm}$ thick silica, using plasma-enhanced chemical vapor deposition (PECVD) at $400^{\circ} \mathrm{C}$ (Centura P5200).

For the characterization, the devices are arranged in a cascade configuration, where each "weak output" is measured and the "strong output" is connected to the subsequent A-MMI. Figure 2 shows the cascade arrangement and a SEM image of a single A-MMI. Therefore, using a nonlinear regression process, the output ratios $(R)$ are calculated for each version of the device in the wavelength range $1540-1580 \mathrm{~nm}$, through the use of a tunable laser (SANTEC TSL 210-F). The results are summarized in Figs. 3 and 4.

Figure 3 shows a discrepancy in the experimental values, which exhibit a variation of up to $\sim 3 \%$ above the simulation results. This trend can be ascribed to the acceptable fluctuations due to possible minor deviations on the device fabrication process and data analysis. Moreover, Fig. 3(b) presents the behavior at $1.55 \mu \mathrm{m}$, from which we can observe the quasi-asymptotic dependence of the splitting ratio for values of the angle $\theta$ below $75^{\circ}$. This implies some limits to the achievable values of $\mathrm{R}$, although power splitting ratio values ranging from $15 / 85$ to
$40 / 60$ can readily be obtained. Interestingly, regardless of the fact that the multimode cavity of our asymmetric MMI splitting devices is altered in relation to their 50:50 symmetric counterparts, they keep maintaining the broadband characteristic of the latter [Fig. 3(a)].

Overall, the results show stable power splitting ratio dependence versus wavelength, despite a slight decrease as the wavelength increase. We consider this behavior is caused by the

a)

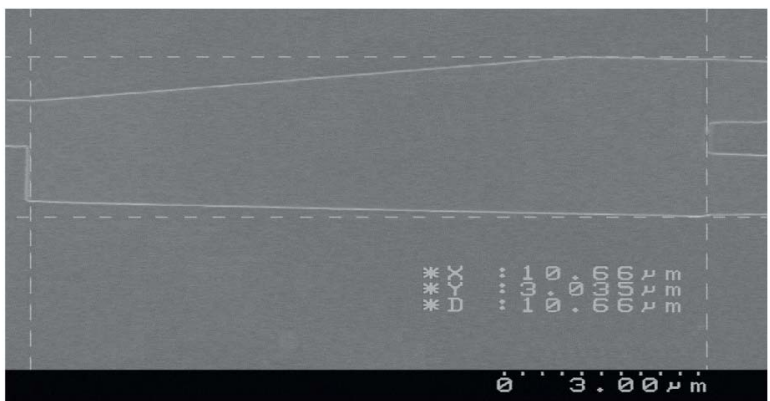

b)

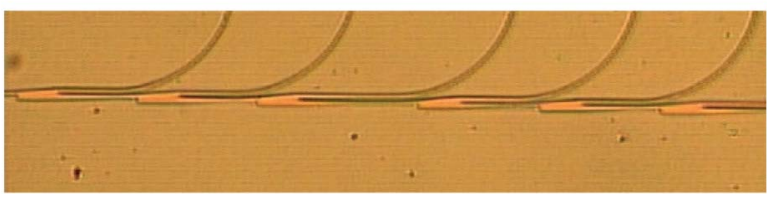

Fig. 2. (a) SEM image of the A-MMI splitter with a ratio of $30 / 70$ $\left(\theta=\sim 83^{\circ}\right)$. (b) Snapshot of the cascade of A-MMI used for the measurements. 
a)

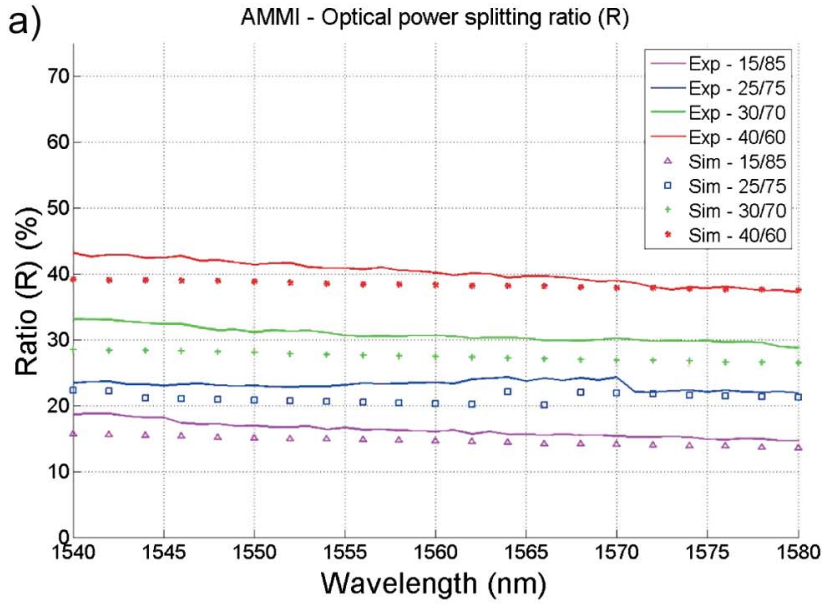

b)

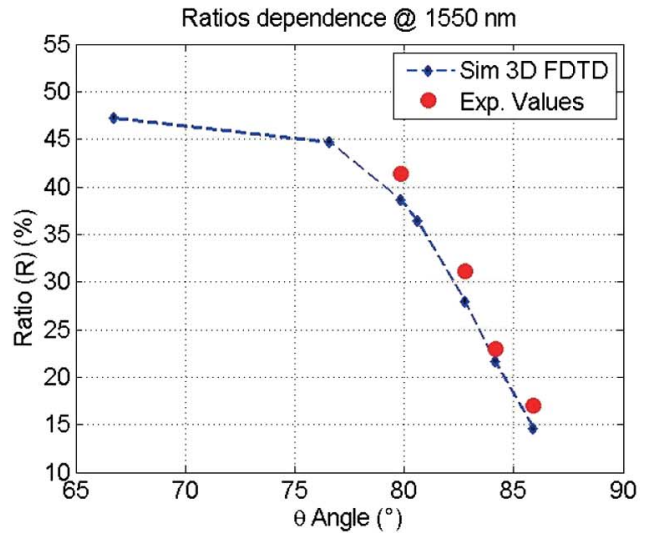

Fig. 3. (a) Wavelength dependence of the $\mathrm{R}$ in the range 1540 $1580 \mathrm{~nm}$, where it is possible to observe broadband of the device. (b) Graph shows a detail of the dependence of the splitting ratio from $\theta$ at $1550 \mathrm{~nm}$. The experimental values deviate of up to a $\sim 3 \%$ from the 3D-FDTD simulations results.

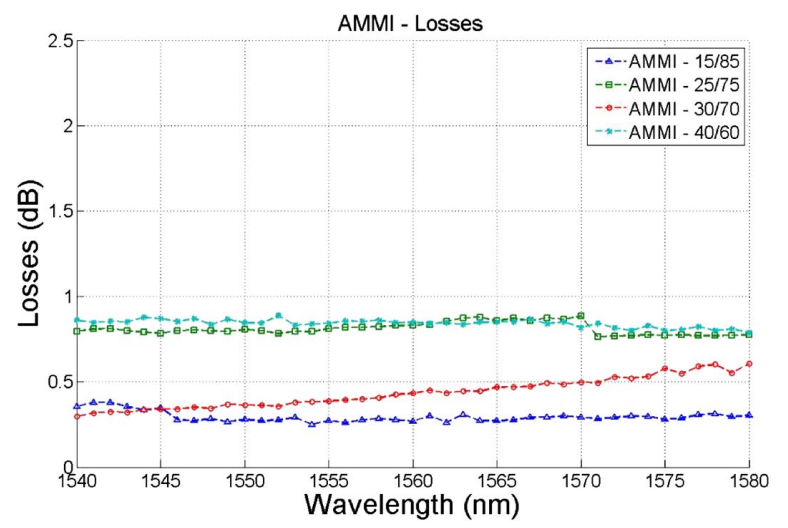

Fig. 4. Wavelength dependence of the device losses in the range $1540-1580 \mathrm{~nm}$. The losses are in the range $\sim 0.4-0.8 \mathrm{~dB}$ for the four considered geometries.

intrinsic nature of multimode interference devices. As reported by Soldano et al. [5], the wavelength causes the interference pattern to change. As a result, the beating length of the device changes and causes the twofold image to move back. However, the presence of edgeless tapers at the outputs helps compensate and, hence, reduces to some extent this slightly decreasing trend.

The overall losses of the A-MMI, as illustrated by Fig. 4, are in the range $\sim 0.4-0.8 \mathrm{~dB}$; this value is slightly higher than that of conventional multimode interference devices [5]. We believe this higher value of losses could be attributed to the higher roughness of the device caused by the breaking of the cavity symmetry or to some minor deviation of the fabrication process, which could cause a systematic error. Further optimization of the fabrication process is expected to reduce these values closer to the theoretical ones $(\sim 0.2-0.3 \mathrm{~dB})$.

In conclusion, we have presented novel A-MMI $1 \times 2$ power splitters featuring arbitrary power splitting ratios in a rib configuration in which the asymmetry is determined by altering the device geometry. As a result, we achieved varying power splitting ratios ranging from $15 / 85$ to $40 / 60$ with relatively low-loss penalty. The dimensions of the proposed device are small $(10.5 \mu \mathrm{m} \times 3 \mu \mathrm{m})$ to minimize the impact on the overall footprint of large scale integrated silicon photonic chips (SiPICs). The measured performances are in line with the conventional symmetric MMI devices in terms of losses and optical bandwidth. Furthermore, the absence of sharp corners in its geometry makes it also more robust than other proposed devices [13]. Overall, our asymmetric MMI power splitting devices show promising characteristics, making them potentially suitable for on-chip power monitoring of large scale Si-PICs.

Funding. 7th European community Framework Programme (FP7-ICT-318240); Spanish Ministry of Science and Technology (TEC2012-38540).

Acknowledgment. The authors thank T. Angelova and L. Bellieres for the fabrication of the device.

\section{REFERENCES}

1. G. T. Reed and A. P. Knights, Silicon Photonics (Wiley, 2008).

2. L. Pavesi and D. J. Lockwood, Silicon Photonics (Springer, 2004), Vol. 1.

3. Z. Zang, T. Minato, P. Navaretti, Y. Hinokuma, M. Duelk, C. Velez, and K. Hamamoto, IEEE Photon. Technol. Lett. 22, 721 (2010).

4. Z. Zang, K. Mukai, P. Navaretti, M. Duelk, C. Velez, and K. Hamamoto, Appl. Phys. Lett. 100, 031108 (2012).

5. L. B. Soldano and E. Pennings, J. Lightwave Technol. 13, 615 (1995).

6. M. Bachmann, P. Besse, and H. Melchior, Appl. Opt. 33, 3905 (1994).

7. G. T. Reed, Y. Hu, D. J. Thomson, A. Z. Khokhar, S. Stanković, C. J. Mitchell, F. Y. Gardes, and G. Z. Mashanovich, Proc. SPIE 9367, 936704 (2015).

8. R. Halir, I. Molina-Fernández, A. Ortega-Moñux, J. G. WangüemertPérez, D.-X. Xu, P. Cheben, and S. Janz, J. Lightwave Technol. 26, 2928 (2008).

9. R. Halir, G. Roelkens, A. Ortega-Moñux, and J. Wangüemert-Pérez, Opt. Lett. 36, 178 (2011).

10. Z. Sheng, Z. Wang, C. Qiu, L. Li, A. Pang, A. Wu, X. Wang, S. Zou, and F. Gan, IEEE J. Photon. 4, 2272 (2012).

11. D. Chack, S. K. Raghuwanshi, V. Kumar, and N. Agrawal, in International Conference on Communication Systems and Network Technologies (CSNT) (IEEE, 2013), pp. 149-151.

12. S. Yi-Ling, J. Xiao-Qing, Y. Jian-Yi, T. Yi, and W. Ming-Hua, Chin. Phys. Lett. 20, 2182 (2003).

13. Q. Deng, L. Liu, X. Li, and Z. Zhou, Opt. Lett. 39, 5590 (2014).

14. "RSoft Design Group, Inc., 200 Executive Group Blvd. Ossining, NY 10562, www.rsoftdesign.com". 